\title{
Clinical treatment of humerus fracture using intramedullary humerus nailing system
}

\author{
Pooja Rawat*, Mohit Kumar, Gaurav Luthra
}

Department of Regulatory, Auxein Medical Pvt. Ltd. Sonipat, Haryana, India

Received: 07 January 2020

Revised: 21 January 2020

Accepted: 22 January 2020

\author{
*Correspondence: \\ Dr. Pooja Rawat, \\ E-mail: clinical2@auxeinmedical.com
}

Copyright: () the author(s), publisher and licensee Medip Academy. This is an open-access article distributed under the terms of the Creative Commons Attribution Non-Commercial License, which permits unrestricted non-commercial use, distribution, and reproduction in any medium, provided the original work is properly cited.

\begin{abstract}
Background: The clinical results of intramedullary humerus nailing system of humeral fracture is controversial variation in implants, and follow up factor and operative technical studies. Humerus fracture is the third long fracture after femoral and tibia bone fracture.

Methods: In this prospective study, 45 patients ( 24 female and 21 male) between the age group of 18-75 years with humerus fracture were taken who did not get conservative management. Type of fracture was categorized according to AO classification. Intramedullary humeral nailing system (compression intramedullary humerus nail, reconstruction nail and reconstruction intramedullary humerus nail) manufactured at Auxein Medical Pvt. Ltd., was used to treatment of humerus fracture. Patient physical fitness was observed according to American Society of Anesthesiologist. Pain scale and outcomes was record from the patients using visual analog scale. Follow up of the patients were taken on 3-week, 8-week, 16-week, 1-year and 2-year.

Results: At 2 years follow up, bone consolidation was present in 44 cases. Only one case has reported of mortality but that was not due to implant related. No clinical and biomechanical complications were reported. Proper healing of implant was achieved.

Conclusions: Intramedullary humeral nailing system is the best treatment option to treat the humerus fracture. Compression intramedullary humerus nail can minimize the gap and increase the biomechanical stiffness.
\end{abstract}

Keywords: Clinical outcomes, Compression intramedullary nailing system, Humerus bone fracture

\section{INTRODUCTION}

A total $4-5 \%$ fracture of humerus bone occurs in proximal humerus seen in accident and emergency department. ${ }^{1}$ About $15 \%$ of them require surgery to treatment. One major problem faced in surgical technique that the high incidence comminuted fractures in elderly patients with osteoporotic bone, not allowing stable internal fixation and only active exercise. The humerus is a long bone in the arm or forelimb that runs from the shoulder to the elbow. It connects the scapula and the two bones of the lower arm, the radius and ulna, and consists of three sections. The humerus bone is the largest bone of human body in forelimb. The end toward the shoulder is called the proximal end. Proximal humerus breaks are generally normal in more elderly people, particularly the elder who have osteoporosis, or weak, permeable bones. The damage frequently happens because of a tumble from a standing stature, when the person grounds on their outstretched arm.

Conservative treatment of humeral shaft fracture is good but has some limitation in some process of soft tissue injury, multiple fracture, and nerve. ${ }^{2}$ Some authors 
speculate that 15 to $64 \%$ of proximal humerus fracture are displaced and may treat by surgical treatment. ${ }^{3}$ The benefits of intramedullary nailing of intense humeral shaft cracks have been bantered since the introduction of this method. Intense cracks of the humerus have a decent mending inclination with non-operative treatment, on account of the magnificent blood supply of the encompassing muscles; in this manner, functional bracing is as yet the treatment of decision in numerous injuries focuses. ${ }^{4,5}$

Consequently, the wide variety of implant is available to treatment the humerus fracture, but to date there is no consensus as to the optimal method of reduction and fixation. Intramedullary humeral nailing system were introduced to reproduce the success seen with similar devices used in the lower extremities. In the intervening period, progress in implant design, the possibility of antegrade insertion, improved rotational stiffness and growing experience in surgical technique have led to better results.

The main concern of this study is to clinical output of Intramedullary humeral nailing system (compression intramedullary humerus nail, reconstruction nail and reconstruction intramedullary humerus nail) in treatment of humerus fracture.

\section{METHODS}

A perspective study was conducted at Mesoamerican University, Quetzaltenango, Guatemala from July 2016 to October 2018, prospective data were collected for patients who received intramedullary humerus nailing system. A total of 45 consecutive patients meeting the inclusion/exclusion criteria were included in this study the mean age at the time of operation was 40.2 years (range 18-75 years). The indications for surgery were humerus fracture. intramedullary humerus nailing system (compression intramedullary humerus nail, reconstruction nail and reconstruction intramedullary humerus nail) (Auxein Medical Private Limited, Sonipat, Haryana, India) were used for Humerus bone union. Patient physical fitness was also observed through America Society of Anesthesiologist (ASA) Grade before undergoing surgery. ${ }^{6}$ Pain scale record from the patient using visual analog scale (VAS). ${ }^{7}$ Follow up of the patient were taken on 3-week, 8-week, 16-week, 1 year and 2 year.

Intramedullary humeral nailing system (compression intramedullary humerus nail, reconstruction nail and reconstruction intramedullary humerus nail) (Figure 1-3) is made of Titanium alloy (Ti6Al4V) and stainless steel (316L), and $4.5 \mathrm{~mm}$ locking bolt, $3.5 \mathrm{~mm}$ locking bolt, and $4.5 \mathrm{~mm}$ proximal screw for compression intramedullary humerus nail were used for fixation of implant.
The surgery was performed under general anesthesia. Clinical results were introduced through the 10-point scale info system named as VAS score. By using radiography (X-ray), the performance of implant was evaluated. Implant shows the good position after 2 year, observing by X-ray. X-ray report were used for inspected bony union, non-union, implant failure and plate migration.

\section{Inclusion criteria}

Patients of both male or female, skeletally mature patient of above 18 years and humerus bone fracture were included in the study.

\section{Exclusion criteria}

Subject having any neuromuscular disorder which would create an unacceptable risk of fixation failure or complications in postoperative care, subjects with substance abuse/alcohol issues, subjects who are incarcerated or have pending incarceration, subject having infection local to the operative site, any uncontrolled systemic disease that, in the opinion of the investigator, would preclude participation in the study (e.g., unstable medical status including uncontrolled elevated blood pressure, cardiovascular disease, and glycemic control) or put the subject at risk due to study treatment or procedures, subject with rapid joint disease, bone absorption, osteopenia, and/or osteoporosis and subject having suspected or documented metal allergy or intolerance were excluded.

The data was analysed using Microsoft excel and presented in number and percentages.

\section{RESULTS}

In the study 45 patients operated with intramedullary humerus nailing system. There were 24 females and 21 males. Female patients were more susceptible in the study. The youngest patient in our series was 19 years and the oldest 73 years. The average age of the patient was 40.2 years as shown in (Table 1). In this study, fracture categorization was recorded according to AO/OTA classification into 11-A2, 12-A2, 12-B1, 12-B2 and $12-\mathrm{A} 3$ where 11 patients had 11-A2 type fracture while 9 patients had 12-A2 type fracture and 11 patients had 12-B1 type fracture, 8 patient had 12-B2 type fracture and 6 patient had 12-A3 type fracture in the humerus bone as shown in (Table 2).

Table 1: Demographic data.

\begin{tabular}{|c|c|c|}
\hline \multicolumn{3}{|c|}{ Demography } \\
\hline \multicolumn{2}{|c|}{ Average age (range) } & 40.2 (range, $18-75$ years) \\
\hline \multirow{2}{*}{$\begin{array}{l}\text { Gender } \\
\mathbf{N}(\%)\end{array}$} & Male & $21(46.67 \%)$ \\
\hline & Femal & $24(53.33 \%)$ \\
\hline
\end{tabular}


Table 2: Fracture classification.

\begin{tabular}{|ll|}
\hline Fracture characteristics & $\mathbf{N}(\%)$ \\
\hline $\mathbf{1 1 - A 2}$ & $11(32)$ \\
\hline $\mathbf{1 2 - A 2}$ & $9(32)$ \\
\hline $\mathbf{1 2 - A 3}$ & $6(4)$ \\
\hline $\mathbf{1 2 - B 1}$ & $11(24)$ \\
\hline $\mathbf{1 2 - B 2}$ & $8(8)$ \\
\hline
\end{tabular}

Out of 45 patient 31 fell under the ASA I (a normal healthy patient) classification 12 patients fell under ASA II (a patients with mild systemic disease) classification, and 2 patients fell under ASA III (a patients with severe systemic disease) classification, none of the patient fell in ASA IV (a patient with sever systemic disease that is constant threat to life).

Table 3: VAS score.

\begin{tabular}{|ll|}
\hline Follw-up time & VAS score (\%) \\
\hline 3 week & 54 \\
\hline 8 week & 44.4 \\
\hline 16 week & 34 \\
\hline 1 year & 19 \\
\hline 2 year & 6.2 \\
\hline
\end{tabular}

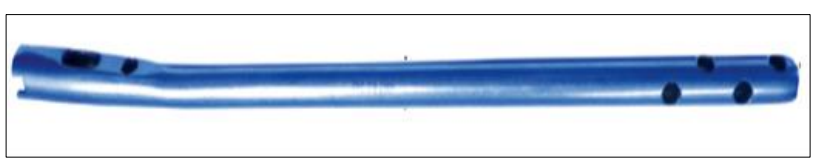

Figure 1: Compression intramedullary humerus nail.

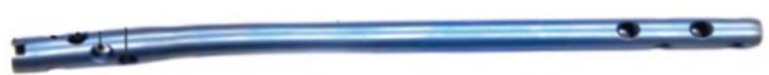

Figure 2: Reconstruction nail.

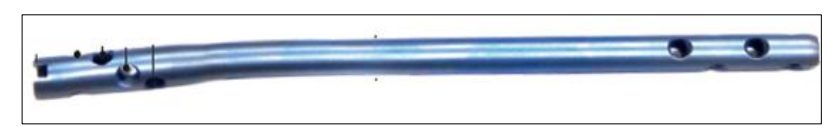

Figure 3: Reconstruction intramedullary humerus nail.

Bone was achieved at 16 week that means 4 months from the day of surgery, it has been confirm through radiological examination by the investigator, as per surgeon clinical union was defined as visible callus bridging the fracture in at least three cortices in radiographs and the patients could use their arms without significant pain or weakness. Pain was recorded for each patient at their respective visits through VAS score, the average pain scale at 3 week was 5.4, 4.44 in 8-week, 3.4 in 16-week, 1.9 in 1 year and 0.62 after 2 year as shown in (Table 3).

Intravenous antibiotics with first generation were given both before and after operation. For open fractures, the antibiotics were given until the wound was clean. Heat packing with hand of movement exercise twice a day was suggested. The active muscle strengthening exercise was increased slowly day by day as per the radio graphical finding. No clinical and biomechanical complication were reported. Proper healing was archived.

\section{DISCUSSION}

Intramedullary humeral nailing system uses as a treatment of humerus bone fracture. In traditionally management of fracture of humerus shaft, often gives the satisfactorily results, but in some cases requires operative stabilization to depict the satisfactorily results. The patients and surgeon prefer the surgery because of rapidly relief. The invention of newer techniques has made the surgery easier to perform with lesser complications. Plate osteosynthesis has high accuracy but requires extra dissection with the risk of damage the nerve and refracture after implant removal.8 but the use of unlocked flexible nails has been complicated by poor rotational stability and slipping of the nails causing joint irritation. Locked nailing overcomes these deficiencies, and results in satisfactory therapeutic outcome. It has been considered the treatment of choice in humeral shaft fractures in the recent past. ${ }^{9}$ Thus, we evaluated the clinical outcome and advantages of intramedullary nailing in our patients with the VAS score. In our study of 45 patients, 46.67 were men and mean age was 40.04 years; All these are attributable to the higher physical activity in these patients. Males are more prone to have humeral shaft fractures but few studies did not find any significant gender difference. Road traffic accident was the most common mode of injury in most of the studies.

In 2002, Cuny et al wrote about the utilization of the Telegraph nail for proximal humerus fracture. Rather than previous nail structures and strategies, this was straight, and the creators prescribed an anterolateral approach through the average and well-vascularized some portion of the cuff. These creators additionally revealed the consequences of the initial 64 nails embedded during the first year (1998-1999). Results were "good," including those with 3-and 4-part fracture. ${ }^{10}$ Srám et al depicted advantages of the Targon PH long nail (Aesculap) that joined the long nail stabilize with angle stable screw proximally their clinical follow-up study in 2007. ${ }^{11}$

Utilizing the statistic information of patients, there is a medium named as VAS score that can choose the standard of careful outcomes and the factor to be adjusted to make it effective. Considering pain scores and looking at the VAS result of various investigations that incorporates statistic information just as biomechanics under controlled preliminaries can be adequate to prescribe the treatment plans. ${ }^{12}$ As for the present study the trial was conducted after every year and the outcome was presented by calculating the VAS score. This has shown good acceptance outcomes. Using the present 
study represents the treatment of humerus fractures surgically by using intramedullary humerus nailing system (compression intramedullary humerus nail, reconstruction nail and reconstruction intramedullary humerus nail), which are designed and manufactured by Auxein Medical Pvt. Limited. No complications found in this study.

\section{CONCLUSION}

Upgrades in implant design and proximal humeral nailing systems keep on advancing. The essential standards include crack decrease to reestablish anatomic connections, strength as required by the nature and the location of fracture, blood supply, and early activation. Now presently proximal humeral nail design and strategy adhere to these standards and may prompt improved results in the treatment of these fractures.

Funding: No funding sources

Conflict of interest: None declared

Ethical approval: The study was approved by the institutional ethics committee

\section{REFERENCES}

1. Horak J, Nilsson BE. Epidemiology of fracture of the upper end of the humerus. Clin Orthop. 1975;112:250-3.

2. Sheriff F. The outcome of interlocking nailing of humeral shaft fracture, a one-year hospital-based study. IJOS. 2018;4(2):95-9.

3. Aaron D, Shatsky J, Paredes JC, Jiang C, Parsons BO, Flatow EL. Proximal humeral fractures: internal fixation. Instr Course Lect. 2013;62:143-54.

4. Bleeker WA, Nijsten MW, Ten Duis HJ. Treatment of humeral shaft fractures related to associated injuries. A retrospective study of 237 patients. Acta Orthop Scand. 1991;62:148-53.
5. Wallny T, Westermann K, Sagebiel C, Reimer M, Wagner UA. Functional treatment of humeral shaft fractures: Indications and results. J Orthop Trauma. 1997;11:283-7.

6. Daabiss M. American society of anesthesiologists physical status classification. Indian $\mathrm{J}$ Anesthesia. 2011;55(2):111-5.

7. Delgado DA, Lambert B, Boutris N. Validation of digital visual analog scale pain scoring with a traditional paper-based visual analog scale in adults. JAAOS Global Res Reviews. 2018;2(3).

8. Lin J, Shen PW, Hou SM. Complications of locked nailing in humeral shaft fractures. J Trauma. 2003;54:943-9.

9. Marchetti PG, Vicenzi G, Impallomeni C, Landi S, Surdo V. The use of elastic nails for intramedullary fixation of humeral fractures and non-unions. Orthopedics. 2000;23:343-7.

10. Cuny C, Pfeffer F, Irrazi M, Chammas M, Empereur $\mathrm{F}$, Berrichi A, et al. A new locking nail for proximal humerus fractures: the Telegraph nail, technique and preliminary results. Rev Chir Orthop Reparatrice Appar Mot. 2002;88:62-7.

11. Srám J, Lukás R, Krivohlávek M, Taller S. Application of the Targon PH long nail in storey fractures and metaphyseal fractures of the proximal humerus. Rozhl Chir. 2007;86:254-62.

12. Zarezadeh A, Mamelson K, Thomas WC, Schoch BS, Wright TW, King JJ. Outcomes of distal humerus fractures: What are we measuring? Ortho Traumatol Surg Res. 2018;104(8):1253-8.

Cite this article as: Rawat P, Kumar M, Luthra G. Clinical treatment of humerus fracture using intramedullary humerus nailing system. Int J Res Orthop 2020;6:266-9. 